\title{
Intrathoracic Manifestations of IgG4-Related Disease
}

\author{
Sian Yik Lim MD, Deepa Panikkath MD
}

\begin{abstract}
Intrathoracic involvement with IgG4-related disease (IgG4-RD) is frequently overlooked in IgG4-related disease patients. In this article we review the intrathoracic findings of IgG4-RD which are variable and protean. IgG4-related disease has been reported to affect the lung parenchyma, pleura, mediastinal/hilar lymph nodes, vasculature, and pericardium within the thorax. Mediastinal and hilar lymphadenopathy is the most common intrathoracic manifestation of IgG4-RD. Four main patterns of pulmonary disease have been described, including the solid nodular type, the bronchovascular type, the alveolar interstitial type, and the round shaped ground glass type. When feasible, a biopsy should be obtained to confirm the diagnosis. Most lesions show characteristic pathologic findings of IgG4-RD: dense lymphoplasmacytic infiltrate, storiform fibrosis, and obliterative phlebitis. While this helps establish the diagnosis, the interpretation of pathology findings in the clinical context is key in making an accurate diagnosis. Mimickers of IgG4-RD should be ruled out, before making a diagnosis. The intrathoracic findings of IgG4-RD can be treated effectively with prednisone, but may require additional immunosuppressive therapies, including rituximab.
\end{abstract}

Key words: IgG4 related-disease, IgG4 lung disease, pleural disease, pulmonary disease, review

\section{INTRODUCTION}

IgG4-related disease (IgG4-RD) is a fibroinflammatory condition characterized by tumefactive lesions, a dense lymphoplasmacytic infiltrate rich in IgG4-positive plasma cells, and storiform fibrosis. ${ }^{1}$

The common organs involved in IgG4-RD include the submandibular glands, lymph nodes, orbits,

Corresponding author: Sian Yik Lim MD Contact Information: limsianyik@gmail.com DOI: 10.12746/swrccc2016.0416.217 and pancreas. ${ }^{2}$ However, this disease process has been reported to affect almost every organ system. Intrathoracic involvement in IgG4-RD, the subject of this review, may occur commonly but is frequently overlooked.

There is a wide range in the prevalence of IgG4RD lung and intrathoracic involvement. In a cohort of 248 IgG4-RD patients with baseline and follow-up imaging, Fei et al reported that IgG4-RD intrathoracic findings were seen in $35 \%$ of the patients. ${ }^{3}$ Zen et al found that $14 \%$ of patients (16/114) had lgG4-RD intrathoracic involvement in a cross sectional study 
of these patients. ${ }^{4}$ Fujinaga et al systematically reviewed diagnostic images from 90 patients with autoimmune idiopathic pancreatitis and noted that $51.2 \%$ of the patients had pulmonary findings. ${ }^{5}$ Wallace et al reported that pulmonary involvement occurred in $17.6 \%$ of patients $(22 / 125) .^{2}$ In the previously mentioned study by Zen et al, pulmonary involvement was noted at $9.5 \%(11 / 114)$ of patients. ${ }^{4} \mathrm{~A}$ higher prevalence has been reported in studies in which there was systemic review of all imaging studies, ${ }^{3,5}$ including one study with the goal to identify intrathoracic findings of IgG4-RD. ${ }^{3} \lg G 4-R D$ involvement of the lung is

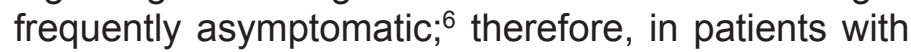
systemic IgG4-RD, intrathoracic/lung involvement may not come to clinical attention, resulting in lower rates of reporting in cross sectional studies. ${ }^{2,4}$

\section{RADIOLOGY}

Pulmonary disease in IgG4-RD has been characterized into four main types based on radiological and pathologic correlations. The four main patterns of pulmonary disease are the solid nodular type, the bronchovascular type (thickening of the bronchovascular bundles and interlobular septa), the alveolar interstitial type (diffuse ground glass opacities, bronchiectasia, and honeycombing), and the round shaped ground glass type (multiple round shaped ground glass opacities) ${ }^{7,8}$ Many patients have several patterns of disease, although a predominant pattern may be identified. ${ }^{7}$ The more commonly seen manifestations are nodules (38-50\%), and airway disease consisting of thickened bronchovascular bundles (69\%) and bronchiectasis (54\%). The frequency of interstitial lung disease is less common. ${ }^{7-10}$

Besides these four main patterns affecting the lungs, airspace disease and central airway involvement have been reported. Airspace disease is characterized by alveolar consolidation with air bronchograms, representing organizing pneumonia. ${ }^{11-13}$ In two separate case reports, these findings were found to improve with steroid treatment. ${ }^{12,13}$ Central airway involvement is characterized by tracheobronchial stenosis. ${ }^{14-16}$ For example, Ito et al reported a case of tracheobronchial stenosis in an autoimmune pancreatitis patient, with bronchoscopy demonstrating fibro- sis with inflammatory infiltrates of plasma cells and lymphocytes. ${ }^{14}$ This was followed by a case series by Yamamoto et al of central airway disease in six IgG4-RD patients, characterized by irregular airway stenosis by bronchoscopy, airflow limitation, and histological findings in the bronchi showing dense IgG4positive plasma cell and lymphocyte infiltrates. ${ }^{16}$

Other intrathoracic manifestations include involvement of the pleura, pericardium, vasculature (the aorta, coronary arteries), and hilar and mediastinal lymph nodes. ${ }^{3,5}$ Hilar and mediastinal lymphadenopathy, usually bilateral, is the most common intrathoracic finding and has been reported in 52.9-78.3\% of patients. ${ }^{3,5}$ IgG4-RD pleural disease presents alone or concurrently with parenchymal disease. Previously reported manifestations of IgG4-related pleural disease include pleural nodules, ${ }^{8}$ pleural effusion, ${ }^{17}$ and extensive adhesive fibrinous pleural thickening (focal and diffuse). ${ }^{17}$ Several cases of histologically diagnosed (on autopsy or pericardiectomy) lgG4-RD pericardial disease have been reported as well. ${ }^{18-20}$ lgG4-RD pericardial disease frequently presents as constrictive pericarditis; Pericardial involvement is usually seen as pericardial thickening on CT. ${ }^{18} \operatorname{lgG} 4$ periaortitis and periarteritis is characterized by well circumscribed arterial wall thickening, homogeneous enhancement of late phase of contrast enhanced CT, and luminal changes (mostly dilated and rarely stenotic). ${ }^{21}$ These findings have been reported within the vasculature of the thorax, including the coronary arteries $^{22}$ and aortic arch. ${ }^{21}$

\section{Pathology}

These different radiological findings are a manifestation of a common process, namely lymphoplasmacytic infiltration of IgG4-positive plasma cells with fibrosis in various parts of the lung parenchyma. Pathological examination of pulmonary nodular lesions shows diffuse lymphoplasmacytic infiltration, fibrosis, and eosinophilic infiltration. Similarly, in patients with radiological findings consistent with the bronchovascular type, severe lymphoplasmacytic infiltration with fibrosis was identified in the interlobular septa or peribronchiolar interstitium. ${ }^{7}$ The radiological and corresponding pathological findings in two patients with 
pulmonary IgG4-RD are shown in Figure 1-4. These images were obtained from two open access case reports published by Kitada et al and Choi et al. ${ }^{17,23}$ Shresta et al reported clinicopathologic features of lung biopsies from six patients with autoimmune pancreatitis and found a lymphangiitic distribution of plasma rich inflammatory infiltrates involving the interlobular septa and visceral pleura. ${ }^{9}$ Dilated lymphatic spaces with increased histiocytes and emperipolesis of lymphocytes were also noted..$^{9}$ A similar lymphangiitic distribution has been reported by several other authors, ${ }^{7,24}$ possibly explaining the radiological findings of the intrathoracic findings in IgG4-RD mimicking sarcoidosis. Obliterative arteritis can be a distinctive feature of IgG4-related lung disease; this is usually not seen in other organs affected by lgG4-RD. ${ }^{8}$
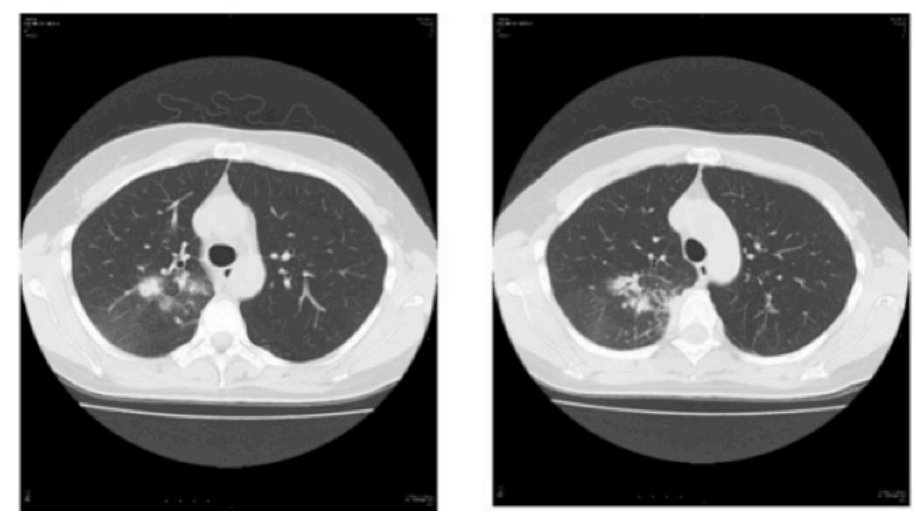

FIGURE 1 Chest CT findings of patient with pulmonary lgG4-related disease

Chest CT revealed a mass density with 12-, 13- and 16-mm spiculations in the S2 segment of the right upper lobe and irregular thickening of the pleura including the paravertebral region.

Pathologically, the pericardial and pleural manifestations of IgG4-RD are characterized by a diffuse fibrinous thickening with a patchy lymphoplasmacytic infiltrate with IgG4 predominance. ${ }^{18-20}$ Periaortitis and periarteritis are characterized as diffuse lymphoplasmacytic infiltration, numerous IgG4-positive plasma cells, and irregular fibrosis of the adventitia. ${ }^{21}$ Pleural effusions have been previously been reported in IgG4-RD and have been described as exudative with a lymphocyte predominance. ${ }^{25,26}$

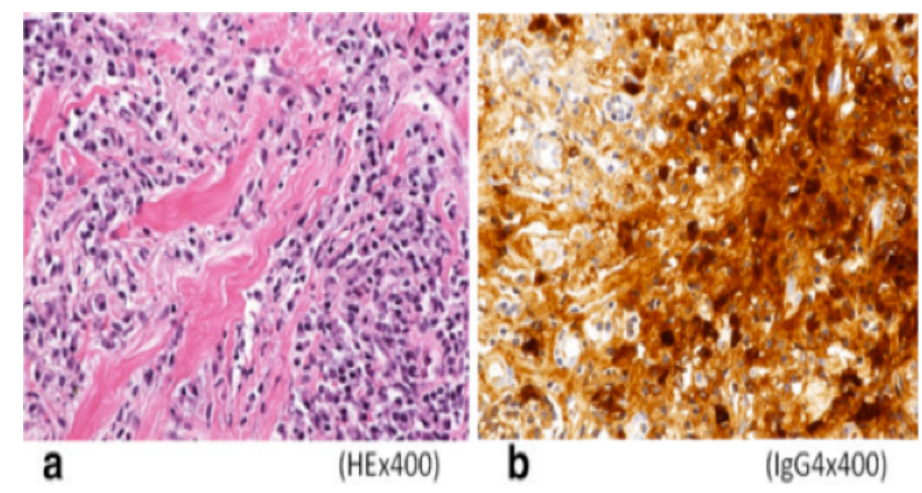

Figure 2 Microscopic examination of surgical resection specimen showing characteristics of IgG4-related disease

a) Pathological examination of the lung revealed marked lymphocytic infiltration in the vicinity of alveolar epithelium free of atypia and marked interstitial connective tissue proliferation with hyaline degeneration.

b) Immunohistochemical examination revealed for IgG4 showed a high IgG4/lgG ratio, exceeding $60 \%$.

Images accessed from: http://www.ncbi.nlm.nih.gov/pmc/articles/PMC3717047/

It is important to keep in mind that because histopathologic findings seen in IgG4-RLD are fairly common in lung sections affected by severe infection and organizing injury from other causes. High lgG4 plasma cell counts and lgG4: IgG ratios have been observed in various conditions, such as multicentric Castleman's disease, rheumatoid synovitis, and granulomatosis with polyangiitis. ${ }^{6,27}$

\section{Diagnosis}

The radiologic and clinical findings of pleuro-pulmonary disease in IgG4-RLD are non-specific and the initial differential diagnosis is broad. Exclusion of malignancy and infection is important because both imaging and pathologic findings from adjacent lung abnormalities of infection and malignancy may mimic non-specific findings of IgG4-RLD. ${ }^{6,27}$ Several mimickers of IgG4-RLD that should be kept in mind when considering the diagnosis of IgG4-RLD include inflammatory myofibroblastic tumors, lymphomatoid granulomatosis, pulmonary hyalinizing granuloma, Rosai-Dorfman disease, multicentric Castleman's 


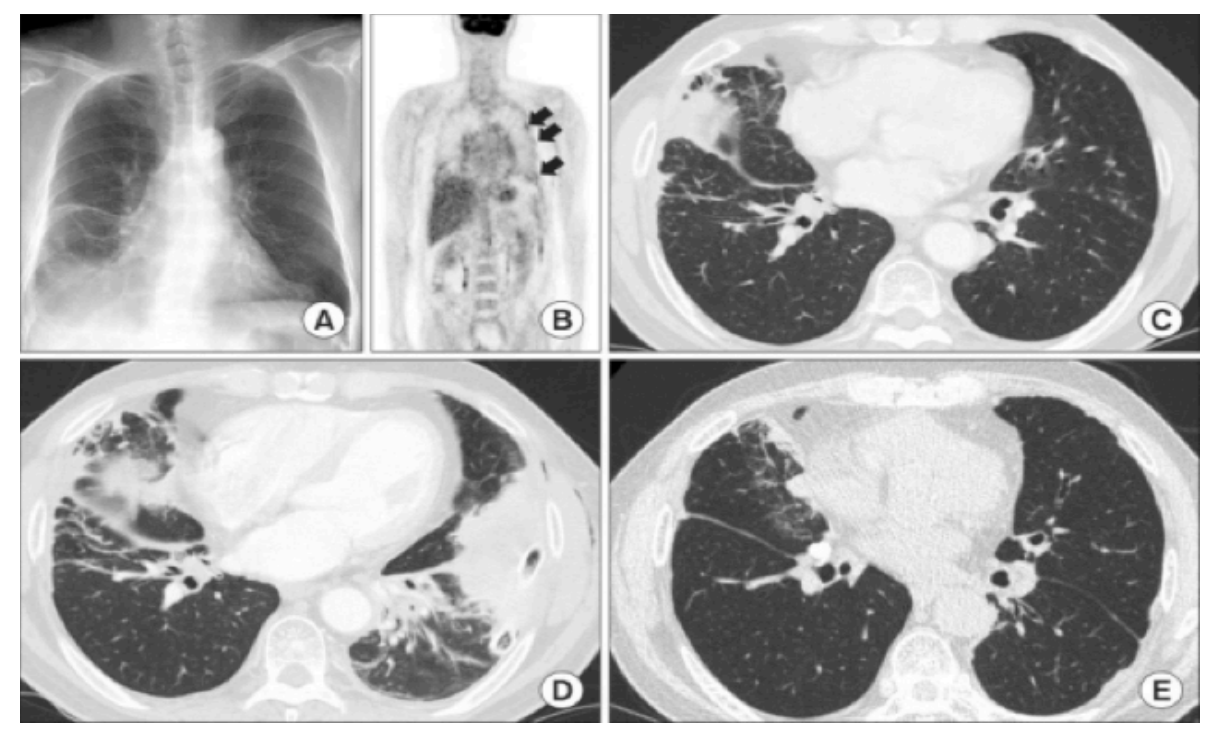

FIGURE 3 Radiologic findings of IgG4-related disease of lung and pleura.

A, C. Chest X-ray (taken 1 year ago) and computed tomography (taken 6 months ago) demonstrate pleural effusion with patchy ground-glass opacity lesions in the right lung.

D. Subsequent computed tomography reveals newly developed, diffuse patchy lesions with pleural thickening and fissural nodularity in both lung, which radiologically suggests mesothelioma or pleural seeding from the lung cancer.

B. These lesions show hot uptake on positron emission tomography.

E. After steroid therapy, the lesions of both lobes are improved with residual small nodule in the left pleura.

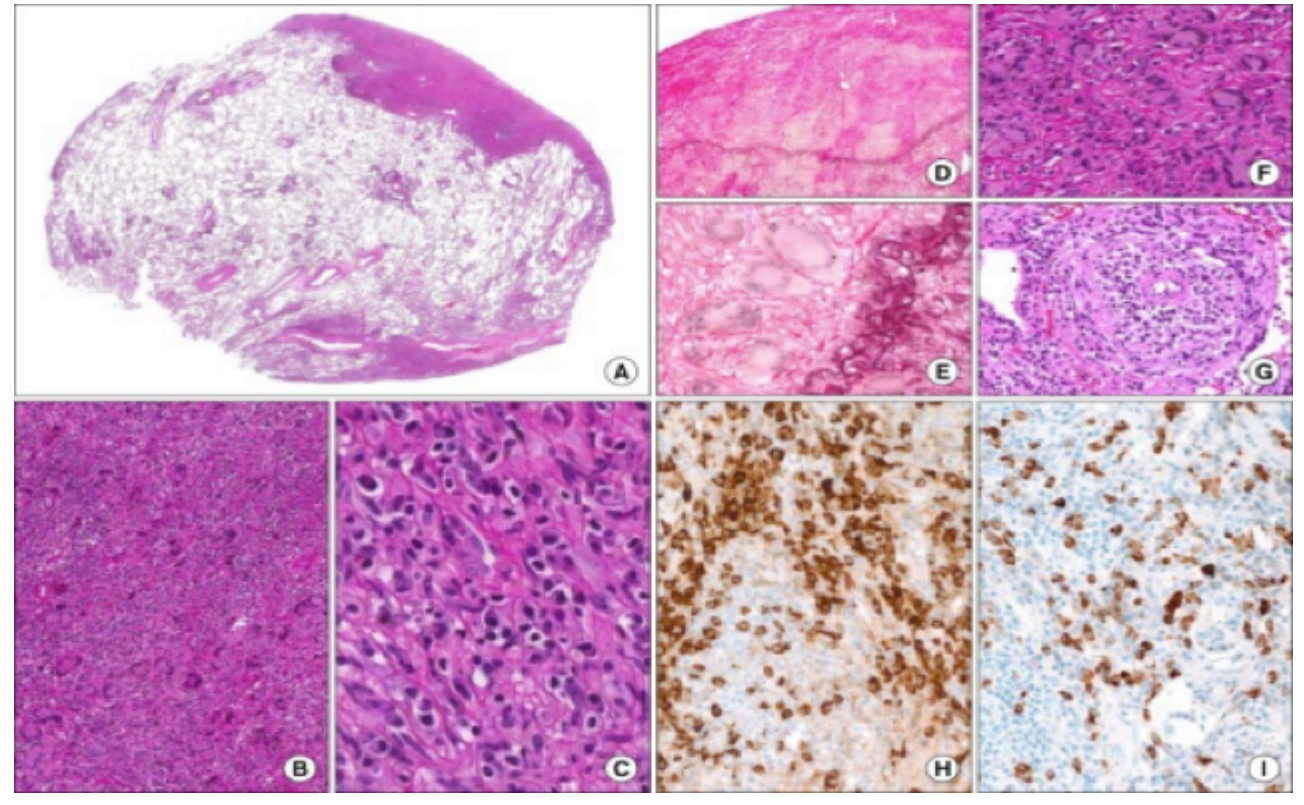

FIGURE 4 Microscopic findings of IgG4-related disease of lung and pleura

A. Scanned view of the lesion displays pleural thickening and subpleural fibrosis. B-D, F, G. Infiltrate cells are mainly composed of lymphocytes and plasma cells in the background of dense fibrosis. Dispersed giant cells are also noted in some areas (C, F, and G, H\&E stain; B and D, Elastic stain; B, ×12.5; C and D, ×200; E, ×100; F, ×40; G, ×400). E. Small blood vessels are infiltrated by lymphoplasma cells without fibrinoid necrosis or granuloma formation. Immunohistochemical staining for $\lg G(\mathbf{H})$ and IgG4 (I) reveal increased ratio of $\lg G 4 / \lg$ positive plasma cells, estimated at about $40 \%(\mathbf{H}$ and $\mathbf{I}, \times 200)$. Images accessed from:

http://www.e-trd.org/DOIx.php?id=10.4046/trd.2014.76.1.42 
disease, lymphoma, granulomatosis and polyangiitis, eosinophilic granulomatosis with polyangiitis and sarcoidosis. ${ }^{6,28}$

In view of the heterogeneity of the pleuro-pulmonary and intrathoracic findings, obtaining a tissue biopsy for the confirmation of the diagnosis of IgG4RLD is crucial. Recently published consensus criteria (Table 1) suggest that lung specimens may be classified as histologically highly suggestive of IgG4-RD when two of the three characteristic histological features (dense lymphoplasmacytic infiltrate, fibrosis, usually storiform, and obliterative phlebitis) are present. Specimens are classified as probable if only one histopathological feature is present. In these cases, elevated IgG4 levels or confirmation of other organ involvement is required. ${ }^{29}$ Interpretation of the histo- pathological findings in the context of the clinical disease is critical in establishing an accurate diagnosis. In a recent study, IgG4 levels had a sensitivity of $90 \%$ in establishing a diagnosis of IgG4-RD. However the specificity of IgG4 levels was only $60 \%$. IgG4 levels can be elevated in other conditions including infections disease, leukemia, and connective tissue disease. ${ }^{30}$

Obtaining lung tissue for a diagnosis may not always be possible because there may be other organs from which tissue can be obtained with less risk. Furthermore, it may be difficult to make a definitive diagnosis based on lung pathology specimens of bronchial thickening and bronchovascular bundle involvement alone because the pathology is less specific and may show only lymphoplasmacytic infiltrate

Table Proposed diagnostic terminology for IgG4-related disease

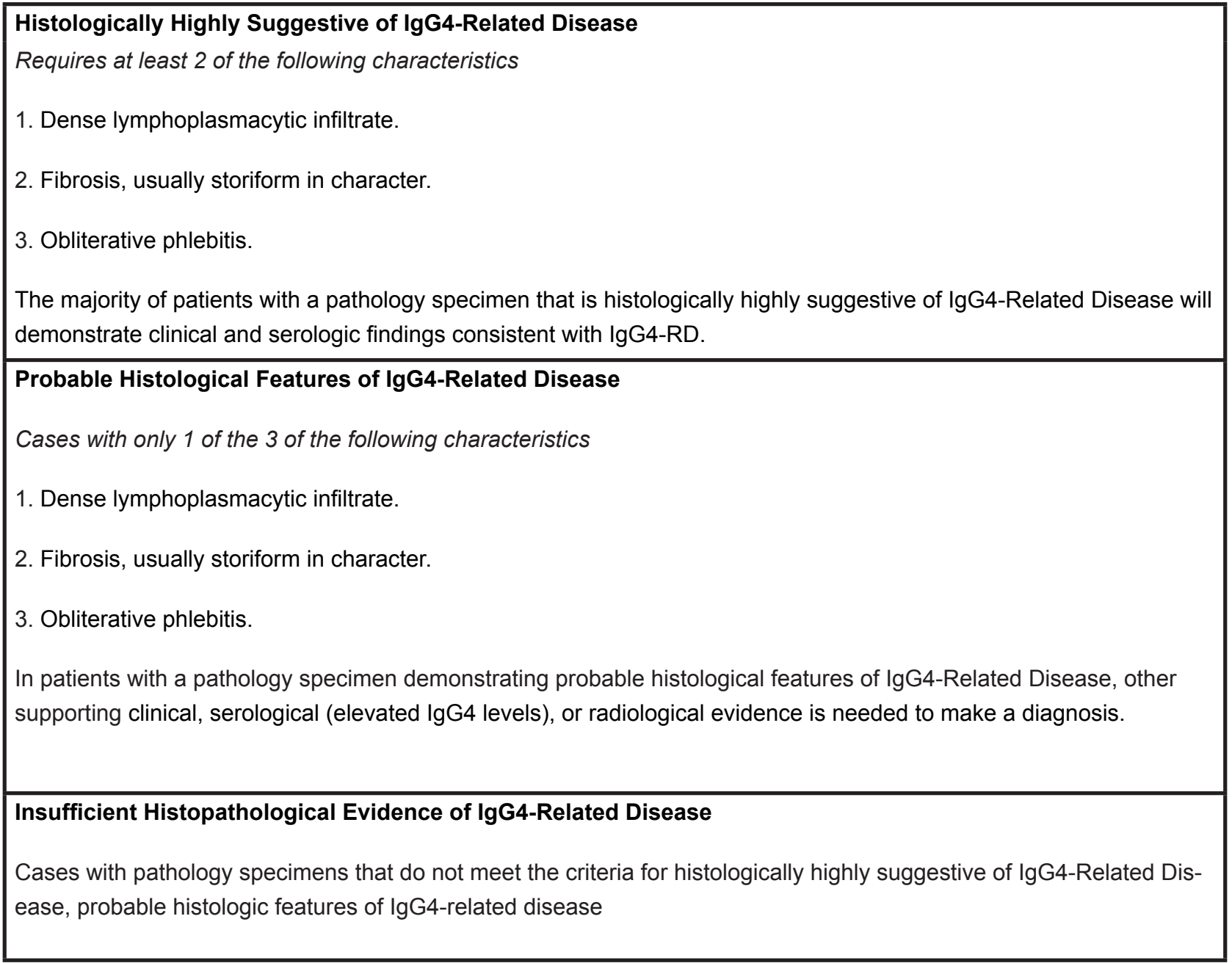


without obliterative phlebitis or storiform fibrosis. ${ }^{28}$ Also, interstitial lesions in IgG4-RLD most commonly demonstrate a non-specific interstitial pneumonia (NSIP) pattern that is nonspecific. ${ }^{9,28}$ Although hilar and mediastinal lymphadenopathy is common, it should be noted that biopsies of the lymph nodes are commonly non-diagnostic because the characteristic storiform pattern of fibrosis is not seen and increased numbers IgG4 plasma cells may be seen in diseases other than IgG4-RD. ${ }^{31}$

\section{TREATMENT}

The intrathoracic findings of IgG4-RD often improve after treatment with prednisone. Fei et al reported that $81 \%$ of the 79 patients with IgG4-RD intrathoracic findings improved with low dose prednisone with or without other immunosuppressant drugs used as maintenance therapy. ${ }^{3}$ Improvement of extra thoracic lesions, including the salivary glands, lacrimal glands, and pancreatitis, was better than intrathoracic lesions. ${ }^{3}$ Zen et al reported that eight of nine patients with the nodular type had resection with no recurrence of pulmonary lesions. One case of the nodular type improved with corticosteroid treatment. Complete resolution or improvement with residual lesions was noted in the bronchovascular type (three of four patients, one patient not treated), round shaped ground glass opacity type (one of one patient), and alveolar interstitial type lesions (two of two patients). In a series of biopsy proven lgG4-RLD reported by Sun, all 14 patients treated with glucocorticoids with or without other immunosuppressant drugs (cyclophosphamide, azathioprine and mycophenolate mofetil) achieved remission of pulmonary disease. Three cases with stable/mild pulmonary involvement were not treated and had a stable clinical course. ${ }^{11}$ Information on the utility of other agents including rituximab in the treatment of IgG4-RLD is sparse.

\section{ConcLusion}

In summary, the intrathoracic presentation of IgG4-RD disease is variable and protean.

Therefore, a high index of suspicion for this diagnosis is important. When feasible, a biopsy should be obtained to confirm the diagnosis. Most lesions show characteristic pathologic findings of IgG4-RD. Clinicians need to interpret the pathology findings and make the diagnosis in the correct clinical context, remembering that other conditions have similar pathological findings.

\begin{abstract}
Author Affiliation: Sian Yik Lim is a fellow in rheumatology in the Division of Rheumatology, Allergy and Immunology, Massachusetts General Hospital, Harvard Medical School, Boston, MA. Deepa Panikkath is a resident in the Department of Internal Medicine, Texas Tech University Health Sciences Center, Lubbock, TX. Both completed their training in June 2016.
\end{abstract}

Received: $04 / 24 / 2016$

Accepted: 10/01/2016

Reviewers: Rishi Raj MD, Anoop Nambiar MD

Published electronically: 10/15/2016

Conflict of Interest Disclosures: None

\section{REFERENCES}

1. Stone JH, Zen Y, Deshpande V. IgG4-related disease. The New England journal of medicine. Feb 9 2012;366(6):539551.

2. Wallace ZS, Deshpande V, Mattoo H, et al. IgG4-Related Disease: Clinical and Laboratory Features in One Hundred Twenty-Five Patients. Arthritis \& rheumatology. Sep 2015;67(9):2466-2475.

3. Fei Y, Shi J, Lin W, et al. Intrathoracic Involvements of Immunoglobulin G4-Related Sclerosing Disease. Medicine. Dec 2015;94(50):e2150.

4. Zen Y, Nakanuma Y. IgG4-related disease: a cross-sectional study of 114 cases. The American journal of surgical pathology. Dec 2010;34(12):1812-1819.

5. Fujinaga $Y$, Kadoya M, Kawa $S$, et al. Characteristic findings in images of extra-pancreatic lesions associated with autoimmune pancreatitis. European journal of radiology. Nov 2010;76(2):228-238.

6. Campbell SN, Rubio E, Loschner AL. Clinical review of pulmonary manifestations of IgG4-related disease. Annals of the American Thoracic Society. Nov 2014;11(9):1466-1475. 7. Inoue D, Zen Y, Abo H, et al. Immunoglobulin G4-related lung disease: CT findings with pathologic correlations. Radiology. Apr 2009;251(1):260-270. 
8. Zen Y, Inoue D, Kitao A, et al. IgG4-related lung and pleural disease: a clinicopathologic study of 21 cases. The American journal of surgical pathology. Dec 2009;33(12):1886-1893.

9. Shrestha B, Sekiguchi H, Colby TV, et al. Distinctive pulmonary histopathology with increased IgG4-positive plasma cells in patients with autoimmune pancreatitis: report of 6 and 12 cases with similar histopathology. The American journal of surgical pathology. Oct 2009;33(10):1450-1462.

10. Tsushima K, Tanabe T, Yamamoto H, et al. Pulmonary involvement of autoimmune pancreatitis. European journal of clinical investigation. Aug 2009;39(8):714-722.

11. Sun X, Liu H, Feng R, et al. Biopsy-proven IgG4-related lung disease. BMC pulmonary medicine. 2016;16(1):20.

12. Duvic C, Desrame J, Leveque C, Nedelec G. Retroperitoneal fibrosis, sclerosing pancreatitis and bronchiolitis obliterans with organizing pneumonia. Nephrology, dialysis, transplantation : official publication of the European Dialysis and Transplant Association - European Renal Association. Sep 2004;19(9):23972399.

13. Taniguchi T, Hamasaki A, Okamoto M. A case of suspected lymphocytic hypophysitis and organizing pneumonia during maintenance therapy for autoimmune pancreatitis associated with autoimmune thrombocytopenia. Endocrine journal. Aug 2006;53(4):563-566.

14. Ito M, Yasuo M, Yamamoto $H$, et al. Central airway stenosis in a patient with autoimmune pancreatitis. The European respiratory journal. Mar 2009;33(3):680-683.

15. Yamamoto H, Yasuo M, Nomura Y, et al. IgG4-related airway involvement which developed in a patient receiving corticosteroid therapy for autoimmune pancreatitis. Intern Med. 2011;50(24):3023-3026.

16. Yamamoto H, Yasuo M, Ito $M$, et al. Clinical features of central airway involvement in autoimmune pancreatitis. The European respiratory journal. Nov 2011;38(5):1233-1236.

17. Choi IH, Jang SH, Lee S, Han J, Kim TS, Chung MP. A Case Report of IgG4-Related Disease Clinically Mimicking Pleural Mesothelioma. Tuberculosis and respiratory diseases. Jan 2014;76(1):42-45.

18. Mori K, Yamada K, Konno T, et al. Pericardial Involvement in IgG4-related Disease. Intern Med. 2015;54(10):1231-1235.

19. Yanagi H, Yamazaki I, Shimizu S, Himeno H, Suzuki S, Masuda M. Constrictive pericarditis caused by immunoglobulin G4-related disease. The Annals of thoracic surgery. Mar 2014;97(3):e71-74.

20. Sekiguchi H, Horie R, Suri RM, Yi ES, Ryu JH. Constrictive pericarditis caused by immunoglobulin G4-related disease. Circulation. Heart failure. Mar 1 2012;5(2):e30-31.

21. Inoue $\mathrm{D}$, Zen $\mathrm{Y}$, Abo $\mathrm{H}$, et al. Immunoglobulin G4-related periaortitis and periarteritis: CT findings in 17 patients. Radiology. Nov 2011;261(2):625-633.

22. Keraliya AR, Murphy DJ, Aghayev A, Steigner ML. IgG4Related Disease With Coronary Arteritis. Circulation. Cardiovas- cular imaging. Mar 2016;9(3):e004583.

23. Kitada M, Matuda Y, Hayashi S, et al. IgG4-related lung disease showing high standardized uptake values on FDG-PET: report of two cases. Journal of cardiothoracic surgery. 2013;8:160. 24. Deshpande V, Chicano S, Finkelberg D, et al. Autoimmune pancreatitis: a systemic immune complex mediated disease. The American journal of surgical pathology. Dec 2006;30(12):15371545.

25. Kojima M, Nakazato Y, Kaneko Y, Sugihara S, Masawa N, Nakamura N. Cytological findings of IgG4-related pleural effusion: a case report. Cytopathology : official journal of the British Society for Clinical Cytology. Oct 2013;24(5):338-340.

26. Yamamoto H, Suzuki T, Yasuo M, et al. IgG4-related pleural disease diagnosed by a re-evaluation of chronic bilateral pleuritis in a patient who experienced occasional acute left bacterial pleuritis. Intern Med. 2011;50(8):893-897.

27. Yi ES, Sekiguchi H, Peikert T, Ryu JH, Colby TV. Pathologic manifestations of Immunoglobulin(Ig)G4-related lung disease. Seminars in diagnostic pathology. Nov 2012;29(4):219-225.

28. Stone JH, Umehara H, Okazaki K. IgG4-related disease. Tokyo: Springer; 2014.

29. Deshpande V, Zen Y, Chan JK, et al. Consensus statement on the pathology of IgG4-related disease. Modern pathology : an official journal of the United States and Canadian Academy of Pathology, Inc. Sep 2012;25(9):1181-1192.

30. Carruthers MN, Khosroshahi A, Augustin T, Deshpande V, Stone JH. The diagnostic utility of serum IgG4 concentrations in IgG4-related disease. Annals of the rheumatic diseases. Jan 2015;74(1):14-18.

31. Martinez LL, Friedlander E, van der Laak JA, Hebeda KM. Abundance of IgG4+ plasma cells in isolated reactive lymphadenopathy is no indication of IgG4-related disease. American journal of clinical pathology. Oct 2014;142(4):459-466. 